\title{
Using Service Learning in an Engineering Program in Ghana
}

\author{
Willie K. Ofosu \\ Associate Professor, School of Engineering Design, \\ Technology, and Professional Programs \\ Penn State Wilkes-Barre \\ Lehman, PA 18627 \\ Email: wko1@psu.edu \\ Francois Sekyere \\ M. Sc. Candidate, Department of Electrical and Computer Engineering \\ Kwame Nkrumah University of Science and Technology \\ Kumasi, Ghana. \\ Email: fsekyere@gmail.com
}

\begin{abstract}
For years, some African universities followed the British education system, but this is gradually changing. For example in Ghana, the three terms to the year approach has been changed to the two semester approach. Also, local conditions and the service learning approach are being included in the curricula. This is being done in the Telecommunications Engineering program at Kwame Nkrumah University of Science and Technology. The program is planned such that some student projects for which the students will earn credits will result in products that will supply community needs. The program is therefore community-centered and provides community service. One project using this approach is based on Broadband Powerline Communication (BPL). BPL can be used in electronic learning at schools and to disseminate information to the community at large. It will empower the people and lead to improving their standard of living. It can also help the nation to become a full participant in the global community, while benefitting from the global economy. This paper describes how this project was designed to suit service learning through an experiential teaching method.
\end{abstract}

Index Terms - Community service, student project, Internet system, service-learning, developing nations, technology divide.

\section{INTRODUCTION}

In a developing nation, the divides between extremes such as the rich and the poor, the educated and the less educated can be glaringly obvious. For instance in Ghana, politicians are adding this to their political platform (Lartey-Adjei, 2008). Such extremes however can provide avenues through which service can be rendered to the community. In this sense, social responsibility and the desire to care for those who need assistance are the attributes that drive and sustain the impulse to provide service. In a developing nation the service can grow beyond the bounds of a local community to involve the whole nation (Osafo, 2007).

Ghanaian Universities draw their student populations from across the whole nation and sometimes beyond. The varied backgrounds of students from urban and rural areas form the basis of discussion on the different rates of development in the different regions within the country. Such discussions naturally lead to further discussion on the technology divide between 
developed and developing nations. While it is necessary to improve technical development across the whole nation, the need is equally great, if not more so, to close the technology divide between the developing nations and developed nations (GNAa, GNAb). Being able to do so in Ghana will naturally make the nation a full participant in the global community, and hence help the nation to take advantage of the global economy (GNAc).

Education is a time tested process that has proved effective in helping nations advance and one approach that is based on current technological development is electronic learning ( $e$ learning) (Martens et al, 2004; McGourty, 2000). The Internet supports this mode of learning where students can receive lessons in a remote setting with the instructor in a different location. This adds to the use of electronic based facilities such as CD in the classroom environment. This is emphasized by the government's interest to promote Information and Communication Technology ICT in schools (GNAd). The point to note is that $e$-learning offers students the opportunity to access educational material by use of the computer, and allows each individual to learn at his or her own pace and also do extra work on their own.

In the Ghanaian environment, the Internet can be deployed in one of two ways. One is Broadband Powerline Communication (BPL) in which data is transmitted on the power line (Amirshahi et al, 2005). This is a viable approach because Ghana has an electrical grid that covers most of the country. This technology will ensure that most of the schools in the nation can have access to the educational programs that may be transmitted at any given period. The other is by wireless, and this will ensure that all parts of the nation can be reached through the radio space. Whichever approach is taken, provision of the equipment will require an initial capital outlay.

The appropriate teaching content is always an issue in education, and one concept is to write the curricula such that it would incorporate ideas that the students will find easy to identify with. In other words, the content should be based on the environment in which the student lives (NEETF Report, 2000). It may be argued that the students will find the material useful if it pertains to them and that would make the learning process easier as the content will not appear to be abstract. The African environment is replete with objects, events and concepts that the students will be aware of in their daily lives and hence involving such material in the classroom will provide a rich local content that will induce the students to learn.

\section{CONCEPT FOR STUDENT PROJECT}

In discussing the environment and its needs with a class of M.Sc. candidates, suggestions for solving some of the local problems were generated. One was an idea for research on the BPL technology that has been developed into a student project. As previously stated, BPL is based on the power grid and this infrastructure is already in operation, provided by Volta River Authority (VRA) covering both urban and rural areas. To achieve the same coverage with cable, DSL or wireless will be extremely expensive financially, and to use satellite will be equally expensive. These other applications are all in their infancy in Ghana hence the infrastructure to support any of them is not as advanced as is the national grid. Even though electrification is well established in Ghana, it is still being further improved (GNAe).

Compared to other technologies BPL Communication is relatively new but it is ideal for a developing country in which the national grid is uniform and is controlled by one authority. Also an existing power line infrastructure is already familiar to all the people and hence a development based on that will only be an extension of a system that the people are already used 
to, and they will therefore have to adapt only to the addition. This eliminates the initial capital outlay for providing the supporting infrastructure for the BPL system. The initial outlay of capital for the application will thus be for the additions necessary to set up the system, which will be a small percentage of what will be needed to create a new infrastructure.

\section{TECHNOLOGY FUSION}

Three major technologies, electrical power transmission, travelling-wave antenna, and data transmission combine to provide the BPL technology. The primary function of the grid is to supply power to various destinations, and this is done mostly using Medium Voltage (MV) overhead power lines in Ghana. Transmission of broadband over MV overhead power lines has been studied and reported on in the literature (Amirshahi et al, 2005), and the related area of transmission of broadband over Low Voltage (LV) (Amirshahi et al, 2006) has also been studied. Electromagnetic Compatibility (EMC) and Electromagnetic Interference (EMI) are important issues (Amirshahi et al, 2005, 2006) among others, such as how signal is coupled to the lines and the propagation constant of the line itself.

The problem can be described as wave propagation on a transmission line (Pozar, 2005), for which the traveling wave can be analyzed by use of the voltages and currents travelling in opposite directions on the line. Due to the finite conductivity of the line, losses will be observed in the waves that propagate along it. The line will also radiate, as well as absorb, electromagnetic energy, as it behaves like an antenna. It is therefore important to evaluate the electromagnetic effects on the structure.

In transmitting data along any medium, it is essential to ensure that the integrity of the data is not seriously impaired. All sources of electromagnetic interference can adversely impact the transmission as noise (Sklar, 2001). It is therefore important to assess the combined internal and external effect on the transmission. As part of the external (man-made) noise, sources of electromagnetic waves such as radio and TV transmissions will be evaluated. Another form of external interference, atmospheric effects which can be significant along the equator and within the tropics, will also be evaluated. These evaluations will be done by the student based on the advice of the faculty member who supervises the project. Heating effect within the system has to be considered, and in line with this, the high ambient temperature in the environment can add to the problem to be resolved.

\section{SERVICE LEARNING}

The BPL project is intended to serve in providing Internet connectivity across the nation of Ghana, and between Ghana and the outside world. The project has come about as a result of the students' concern for the lack of facilities, such as hospitals, and limited educational facilities in some rural and remote areas of Ghana (GNAc,d). Telemedicine, and Internet in schools for instance will be possible with the deployment of the BPL system. It will count as community service since that is what it is intended to do, and for the student, it will count as a Master's project for which the student will receive a grade towards graduation. Needless to say, the students in this class are already showing a high degree of social consciousness which they demonstrated by their desire to help their communities to develop. The selection of the project demonstrates the awareness of the student in turning his effort into academic gain. With time, 
entrepreneurial considerations may be encouraged to help sustain the developmental steps being taken.

The engineering principles involved in the project are not new to manufacturing or design processes. The environmental conditions and the electromagnetic radiation in the radio space can however impact the performance of the system. These form the local content that needs to be emphasized in the curriculum as they impact the design of the BPL system.

Additive noise can be a major problem (Sklar, 2001) in all electrical and electronic systems, and where there is a high ambient temperature as experienced in the equatorial region, temperature effects can exacerbate the problem of noise. Electromagnetic radiation from sources such as radio and TV transmissions in the region of the system will also contribute to the overall noise problem. Also, possible atmospheric noise may be encountered in the equatorial region and that should be evaluated. The fidelity of the received signal is a function of the type of modulation among other factors such as noise. The receiver will thus have to perform other functions such as filtering and noise suppression, and these factors can be influenced by the conditions in the equatorial region.

In addition to the analytical work that is done, experimental work will be done in collecting data that will confirm solutions derived from analysis. One such experiment is based on the use of an RF Analyzer to collect data on radio and TV broadcast stations in the selected area for experimentation to characterize the electromagnetic radiations present in the radio space at the site. The magnitude of each emission will be determined by use of a hand-held RF Analyzer and the result will indicate the impact, if any, of that particular source as noise as stated above.

Torrential rainfalls are prevalent in the tropics. The aspect of importance here is the level of humidity that is experienced and the effect this will have on the system. Moisture can form a film on circuit boards that can cause a short between components, and this can have disastrous effects. In the case of systems that are in the open, proper precautionary methods have to be employed to ensure that components are protected from the water. Long term effects such as corrosion can also be a source of problem. For a system that is intended to be in service for a reasonable length of time, it is necessary to ensure that such possible sources of error are considered in the design process.

\section{PROGRAM COMPONENTS}

The project is intended for the Masters' level of the Electrical Engineering program with further emphasis on the engineering principles that are directly affected by the equatorial environmental conditions. These conditions will impact the technology. In addition to these, the societal needs that the project can serve have been highlighted. This demonstrates that the project is intended to have an end product that can be deployed to serve a need of the people. This point is highlighted because it provides one of the essentials to the development program of the nation. Once in place, the BPL can be used to disseminate information that can be used by individuals to enhance their standard of living, as well as support the peoples' aspiration towards development. It can also be used to respond to social needs such as telemedicine as stated above. The following flow diagram depicts the format described with the service learning component indicated. 


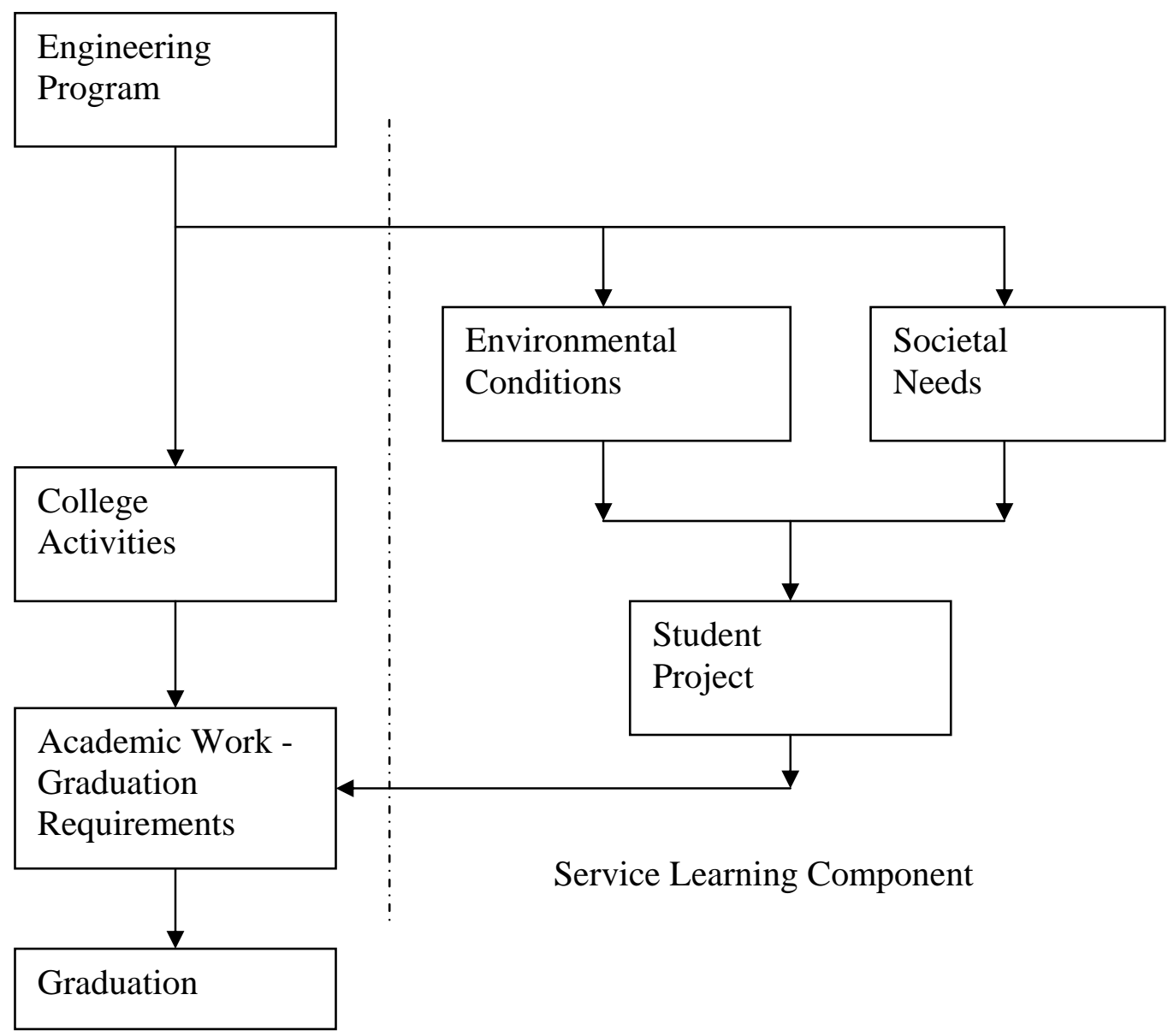

FIGURE 1. FLOW CHART OF PROGRAM COMPONENTS.

The entrepreneurial component that can result from the program for the people in the community is not included in the flow diagram but is also important. Developing a product that can be used as a basis for a business is useful for economic reasons and also because the business can in turn provide some assistance to the program. This can lead to an industry and college partnership out of which many benefits can derive. An advantage to the economic aspect is in the improvement in the lives of those involved in the business.

In a much broader sense, the BPL project can provide connectivity not only to other users in Ghana, but also to the outside world. This is important as Ghana is engaged in becoming a participant in the global community. Setting up the BPL to cover both urban and rural areas in Ghana will ensure that all people in the country have access to the outside world. This will support the developmental objectives of the nation.

\section{GUIDELINES FOR PROJECT SELECTION}

The environment and conditions in the developing country in which the student is working will present certain difficulties. One of these which will present varying degrees of difficulty is travel 
to rural or remote areas. Another is sufficient financial support to procure equipment and components. The faculty member supervising the project has to be conscious of such factors and advise the student accordingly to ensure the student is well prepared and in a position to complete the project. In cases where a grant is needed, the faculty member may assist in seeking the funds.

To cite an example, a grant from Engineering Information Foundation (EiF) based in New York was used to purchase some laboratory equipment for the Telecommunications program. Some pieces of the equipment, for example an RF Analyzer, can also be used in the BPL project.

As stated above, one of the criteria used in the selection of the project was that it should conform to the service learning mode (Cline et al, Spring 2008). Another criterion was that there will be a resultant product that will serve the community. The student therefore has to interact with the community as well as reflect on the worthiness of the contribution to, and support for the progress of the community. A third criterion was that the student will employ knowledge gained from the classroom in completing the project. This makes the project comprehensive, and will test the student's comprehension and skills in the chosen field. The project is sufficiently complex to challenge the student, yet feasible to complete.

The scope of the project in its entirety is rather large. It comprises establishment of its feasibility, conditioning of the power grid for data transmission, and deployment of the application. Conditioning of the power grid and deployment of the application will be through partnership with industry, and for such reasons, KNUST is cooperating with VRA. These two sections are not considered in this paper. The objective at this initial stage of the project is to establish the feasibility and KNUST has been cooperating with VRA in this area as well, since they have authority over the power grid.

The initial stage of the research component of the project is intended to establish the feasibility of the application. This stage consists of activities such as

a) determining sources of radiation and their magnitudes present at the site of experimentation,

b) relating these by their frequencies to applications in the vicinity,

c) determining what radiations, if any, may have been captured by the transmission lines which will manifest as noise, and

d) doing an initial loop-back test.

The first two activities will be done concurrently and after this, the last two activities will follow, also concurrently. Other activities will cover topics such as rate of transmission, latency, throughput and channel capacity. Also, possible improvement of the data rate will be considered. At this stage there is only one student who is at the Masters' level of his studies in Electrical Engineering working on the project and it is not intended for him to complete all these activities. A good start in these activities can establish that the BPL application is possible, and this is expected of the student. The project component of the Masters' program is expected to cover twelve months. A guideline of timeline suggested to the student is to use from six to eight months for the experimental and mathematical analysis of the project and the rest of the time to write a project report. 
Evaluation will be based on the student's performance, and this will involve his mastery of the subject area and related areas, execution of the project, time management and interpersonal skills in communicating with the members of the community, and assessment of the submitted report.

\section{CONCLUSION}

Evaluation of various communities' needs resulted in suggestions of ways to respond to the needs. These led to a student project in BPL which is being conducted in the service learning mode. This in turn responds to a student's need to advance his academic standing as well as be a productive member of the community. Environmental conditions have been included in the curricula to personalize the curricula for the students in the region and hence minimize the abstraction in the curricula. The BPL project will support $e$-learning and also open up the Ghanaian community to the global community.

\section{REFERENCES}

Amirshahi, P., Kavehrad, M., "Medium Voltage Overhead Power-line Broadband Communications;

Transmission Capacity and Electromagnetic Interference”; Proceedings of ISPLC 2005, Vancouver,

Canada, April 2005, pp 2-6.

Amirshahi, P., Kavehrad, M., "Broadband Access over Medium and Low Voltage Powerlines and use of White Light Emitting Diodes for Indoor Communications,” IEEE Consumer Communications \& Networking Conference, Las Vegas, Nevada, January 2006.

Cline, R. C., Kroth, M., (Spring 2008). “The Challenges of Using Service Learning in Construction Management Curricula” IJSLE Vol.3, No. 1, pp. 1-8, Spring 2008, ISSN 1555-9033

Ghana News Agency (GNAa), “Closing ICT Gap Between Developed and Developing Countries”, www.ghanaweb.com, General News Monday, April 28, 2008

Ghana News Agency (GNAb), “Lifting Information Technology Needed to Close Poverty Gap”, www.ghanaweb.com, General News Thursday, April 242008

Ghana News Agency (GNAc) “Ghana can’t Attain Middle Income Status Without ICT”, www.ghanaweb.com, General News Thursday, May 3, 2007

Ghana News Agency (GNAd), "Ministry to Promote ICT in Schools”, www.ghanaweb.com, General News Friday, December 21, 2007

Ghana News Agency (GNAe) “580 Rural Communities to Benefit from Electrification Project” www.ghanaweb.com, Ghana News Monday, January 7, 2008.

Lartey-Adjei, Festus K., “CPP will push for a Robust District Policy”, www.ghanaweb.com, General News Sunday, April 6, 2008.

Martens, Rob L.; Gulikers, Judith; Bastiaens, Theo; "The Impact of Intrinsic Motivation on e-Learning in Authentic Computer Tasks”, Journal of Computer Assisted Learning, Vol. 20, pp. 368-376, 2004.

McGourty, Jack, “Using Multisource Feedback in the Classroom: A Computer-based Approach”, IEEE Transactions on Education, Vol.43, No.2, pp. 120-124, 2000.

NEETF, “Environmental-based Education: Creating High Performance Schools \& Students” September 2000. 
Osafo, Kweku, "Economic Development Plan for Ghana - Osafo", www.ghanaweb.com, General News Monday, November 5, 2007.

Pozar, D. M., “Microwave Engineering” $3^{\text {rd }}$ Edition, John Wiley, Chp 2, 2005.

Sklar, B., “Digital Communications, Fundamentals and Applications”, $2^{\text {nd }}$ Edition, Prentice Hall, Chp. 1-4., 2001.

\section{ACKNOWLEDGMENT}

The authors wish to give their sincere thanks to Engineering Information Foundation (EiF) for the grant that made it possible to partly supply the Telecommunications laboratory of Kwame Nkrumah University of Science and Technology with equipment. 